\title{
The prognostic value of circulating lymphocyte counts and ABO blood group in lung cancer stereotactic body radiation therapy: a retrospective study
}

\author{
Meng Chen ${ }^{1,2}$, Kuifei Chen ${ }^{1,2}$, Shuling $\mathrm{Li}^{1,2}$, Yinnan Meng ${ }^{2}$, Yangyang Shi ${ }^{3}$, Xiaofeng Chen ${ }^{4}$, Haihua Yang ${ }^{1,2}$ \\ ${ }^{1}$ School of Medicine, Shaoxing University, Shaoxing, China; ${ }^{2}$ Key Laboratory of Radiation Oncology of Taizhou, Radiation Oncology Institute of \\ Enze Medical Health Academy, Department of Radiation Oncology, Taizhou Hospital Affiliated to Wenzhou Medical University, Taizhou, China; \\ ${ }^{3}$ Department of Radiation Oncology, University of Arizona, Tucson, AZ, USA; ${ }^{4}$ Department of Radiation Oncology, Indiana University School of \\ Medicine, Indianapolis, IN, USA \\ Contributions: (I) Conception and design: H Yang, M Chen; (II) Administrative support: H Yang; (III) Provision of study materials or patients: M \\ Chen; (IV) Collection and assembly of data: M Chen, K Chen, S Li; (V) Data analysis and interpretation: M Chen, Y Meng, H Yang; (VI) Manuscript \\ writing: All authors; (VII) Final approval of manuscript: All authors. \\ Correspondence to: Haihua Yang, MD. Key Laboratory of Radiation Oncology of Taizhou, Radiation Oncology Institute of Enze Medical \\ Health Academy, Department of Radiation Oncology, Taizhou Hospital Affiliated to Wenzhou Medical University, Taizhou 317000, China. \\ Email: yhh93181@hotmail.com.
}

Background: Clinically, there is a lack of simple and feasible indicators to predict the efficacy of stereotactic body radiation therapy (SBRT). Circulating lymphocyte counts (CLCs) is considered to be related to curative effect in conventional radiotherapy of lung cancer, and blood groups are also associated with the survival. In this study, we investigate the prognostic value of CLCs and ABO blood groups in lung cancer patients treated with SBRT.

Methods: We retrospectively analyzed 191 patients who were treated with lung cancer SBRT in Taizhou Hospital of Zhejiang Province from September 2014 to December 2018. The medical record system of Taizhou Hospital was used to collect relevant clinical data, such as stage, CLC, ABO blood groups and other important clinical co-variates. The effects of SBRT were evaluated by patient reexamination image data and telephone follow-up. The RECIST 1.1 standard was used to evaluate the short-term efficacy in the first, third, and sixth months after SBRT. Progression-free survival (PFS) was defined as the time from the day of SBRT to disease progression or death from any cause. Overall survival (OS) was measured from the day of SBRT until the last follow-up or death. Survival curves and univariate, multivariate logistic-regression analyses were used to expound the prognostic factors for local control (LC), PFS, and OS of lung cancer SBRT patients.

Results: Univariate and multivariate analysis results showed that post-SBRT CLCs were independent factors for the short-term efficacy 3 and 6 months after lung cancer SBRT [hazard ratio $(H R)=0.249$, $\mathrm{P}=0.037 ; \mathrm{HR}=0.347, \mathrm{P}=0.012]$. Survival analyses showed that the PFS and OS of lung cancer SBRT patients with A blood type was significantly shorter than that in the other three non-A blood groups (PFS: 6.5 vs. 10 months, $\mathrm{HR}=1.535, \mathrm{P}=0.020$; OS: 24 vs. 41 months, $\mathrm{HR}=1.578, \mathrm{P}=0.048$ ). Moreover, the patients with high post-SBRT CLCs in the non-A blood group had the longest PFS and OS after lung cancer SBRT ( $\mathrm{HR}=0.551, \mathrm{P}=0.043)$.

Conclusions: Lung cancer SBRT patients with high-post-SBRT CLCs and non-A blood groups seem to exhibits best curative effect, which represent a potential opportunity to improve the clinical management of these patients. The mechanisms of this association deserve further verification and investigation.

Keywords: Circulating lymphocyte counts (CLCs); ABO blood group; lung cancer; stereotactic body radiation therapy (SBRT); prognostic factors 
Submitted Dec 27, 2021. Accepted for publication Feb 18, 2022.

doi: $10.21037 /$ jtd-22-130

View this article at: https://dx.doi.org/10.21037/jtd-22-130

\section{Introduction}

Lung cancer is the leading cause of cancer-related death worldwide, and its incidence and mortality is steadily rising. Lung cancer accounts for $11.4 \%$ of all cancer cases and $18 \%$ of total cancer deaths globally per year (1). Radiotherapy is a basic treatment method for lung cancer at different stages. Compared with conventional external beam radiation therapy (EBRT), stereotactic body radiation therapy (SBRT), which delivers a high dose in a few fractions, has a better rate of local control (LC) (2,3). In 2018, the median overall survival (OS) of early-stage lung cancer after SBRT was 4 years, and the 5 -year OS was $40 \%$ (4). The efficacy of SBRT was found to be similar to surgery and had fewer adverse impacts (5). Unfortunately, most tumor patients cannot avoid developing local or distant tumor recurrence, with some patients diagnosed in the locally advanced stage or metastasis stage. Providing active local treatment combined with systemic treatment can strengthen LC, improve quality of life, and prolong life (6). However, there are significant differences in the efficacy of SBRT among individuals. The indicators that can predict the efficacy of SBRT in lung cancer are very important for selecting the best treatment plan and follow-up strategy and to improve the treatment effect for individual patients. Routine blood examination is a necessary examination for patients before and after treatment, and blood type is a common clinical examination item. However, whether these two simple and easily available indicators can be used to predict the curative effect of SBRT in lung cancer has not been studied.

Circulating lymphocyte counts (CLCs), which is an important part of blood examination, have a decisive impact on the immune system of cancer patients. Many clinical reports have demonstrated that CLCs were correlated with LC and OS $(7,8)$. Lymphocytes are very sensitive to radiotherapy, which can destroy them at as low as 1 Gy (9). Furthermore, radiation-induced lymphopenia (RIL) was found to be negatively associated with OS and relapse-free survival in multiple cancers including head and neck cancer, lung cancer, breast cancer, and pancreatic cancer (10-12). The irradiation dose, irradiation mode, and irradiation volume correlate with the effectiveness of radiotherapy. In the past, some studies found that SBRT was significantly better than conventional fractionation radiotherapy $(2,3)$. But clinical studies such as RTOG 0617 found that increased conventional fractionation dose had the opposite result (13). These findings show that only increasing the biological dose does not improve the efficacy of radiotherapy. Some studies have found that high dose radiotherapy and a large target area significantly affects lymphocytes immune system $(14,15)$, and McLaughlin et al. [2020] discovered that SBRT reduced RIL in earlystage non-small cell lung cancer (NSCLC) (16). Therefore, the effect of radiotherapy on lymphocytes highlights an advantage of SBRT over conventionally fractionated chemoradiation (CRT). In the past, we also studied the models of the effects of SBRT and CRT on lymphocytes, and we also found that SBRT significantly alleviated the effects of CRT on lymphocytes (17). However, up to now, the ability of lymphocytes to predict the efficacy of lung cancer SBRT has not been reported.

The ABO blood group system is widely used in clinical practice. Some studies have shown that the ABO blood group is associated with a variety of diseases and pointed out that the $\mathrm{ABO}$ blood group may be a risk factor for some cancers (18-20). There are still many doubts about the prognostic value of blood groups. The purpose of our study was to confirm whether the CLCs and blood type have a prognostic value in lung cancer patients treated with SBRT. We present the following article in accordance with the REMARK reporting checklist (available at https://jtd. amegroups.com/article/view/10.21037/jtd-22-130/rc).

\section{Methods}

\section{Patient selection and collection}

The study was conducted in accordance with the Declaration of Helsinki (as revised in 2013). This retrospective study was approved by the ethics committee of Taizhou Hospital of Zhejiang Province (No. K20170320) and individual consent for this retrospective analysis was waived. Relevant clinical data, such as age, gender, smoke, stage, pathology, location, systemic therapy, biological equivalent dose (BED), blood types and other important clinical co-variates, were collected by the medical record 
system. Pre- or post-SBRT CLCs were collected from patient routine blood tests within 1 week before and after SBRT. The inclusion criteria for cases were as follows: (I) lung cancer patients treated with SBRT in Taizhou Hospital of Zhejiang Province between September 2014 and December 2018; (II) patients had a pathological diagnosis of primary and metastatic lesions in the lung; (III) before, during and after treatment, the patient had no infection or other systemic inflammatory symptoms; (IV) the patient had routine blood tests before and within 1 week after treatment; and (V) integrated follow-up data about the patient were available, including LC and OS.

\section{$S B R T$}

All patients were supine positioned with both arms raised above their head. They were immobilized with a thermoplastic body mask. Enhanced four-dimensional computed tomography (4D-CT) scans were obtained in free quiet breathing mode for motion management. In the simulation process, unenhanced CT scans were also acquired for dose calculations at $5 \mathrm{~mm}$ slice thickness by a CT scanner (Discovery CT590 RT, GE). The gross tumor volume was contoured on lung CT windows. The internal tumor volume (ITV) was contoured on the maximum intensity projection phase of the 4D-CT scan and was copied to unenhanced CT images. The planning target volumes were generated by adding to the ITV a $5 \mathrm{~mm}$ margin to correct for positional inaccuracies. Other organs at risk were contoured on unenhanced CT images. Each SBRT plan was delivered with 9- to 11-fixed field coplanar static intensity-modulated radiotherapy. The collapsed cone convolution algorithm was used for dose calculations in the Pinnacle Version 9.10 planning system (Philips Medical Systems, Milpitas, CA, USA). The prescription dose was 50-70 Gy in 5-10 fractions for central and peripheral lung cancer, respectively. The treatment plan was evaluated by a radiation oncologist according to RTOG 0915. Before treatment, each plan was verified to assess dosimetric agreement. Each patient was treated with image guidance at each treatment through cone-beam CT to ensure proper alignment of the geometric treatment center.

\section{Therapeutic effect evaluation}

The first endpoint is the LC rate. The RECIST 1.1 standard was used to evaluate the LC in the first, third, and sixth months after SBRT, which was defined to be the sum of complete response, partial response, and stable disease. When the 3- and 6-month LC after SBRT was difficult to judge by CT scan diagnosis, we sought the help of experienced oncologists. If the consensus as still not reached, some patients underwent a positron emission tomography (PET)-CT for better follow-up treatment. The secondary endpoint was progression-free survival (PFS), which was defined as the time from the beginning of SBRT to disease progression or death from any cause. The third endpoint was OS, which was measured from the beginning of SBRT to the last follow-up or death.

\section{Statistical analysis}

Count data were expressed as a percentage (\%). The lymphocyte values before and after treatment were grouped by the median value. The change of CLCs between the two groups was also grouped by the median value of $0.30 \times 10^{9} / \mathrm{L}$. Survival curves were analyzed by the Kaplan-Meier method and compared by the log-rank test. Univariate and multivariate logistic-regression analyses were used to expound the potential prognostic factors of LC, PFS, and OS in lung cancer SBRT patients. The chi-square test was used to evaluate differences in baseline clinical characteristics among groups. All statistical analyses were performed using SPSS 26.0 (IBM Corp. in Armonk, NY, USA), and two-sided $\mathrm{P}$ values $<0.05$ were considered statistically significant.

\section{Results}

\section{The general clinical features of 191 lung cancer patients}

The data of 191 patients treated with lung SBRT between September 2014 and December 2018 were retrospectively analyzed. Table 1 shows that, among the 191 lung cancer patients, the proportion of male patients $(76.4 \%)$ was more than female patients $(23.6 \%)$, and the median age of all patients was 65 years old. Among ABO blood groups, O-type had the highest proportion, followed by A-type, accounting for $41.4 \%$ and $28.3 \%$ of patients, respectively. The pretreatment and post-treatment total CLCs were obtained and analyzed. Medians were 1.4 and $1.1 \times 10^{9} / \mathrm{L}$. Change medians of the pre- and post-SBRT CLCs were $0.3 \times 10^{9} / \mathrm{L}$. The CLCs counting boundary was defined as the median of post-SBRT CLCs, which was $1.1 \times 10^{9} / \mathrm{L}$. 
Table 1 Clinical characters of 191 lung cancer patients

\begin{tabular}{|c|c|c|}
\hline Characters & Number of cases & Constituent ratio (\%) \\
\hline \multicolumn{3}{|l|}{ Gender } \\
\hline Male & 146 & 76.4 \\
\hline Female & 45 & 23.6 \\
\hline \multicolumn{3}{|l|}{ Age } \\
\hline$<65$ years old & 94 & 49.2 \\
\hline$\geq 65$ years old & 97 & 50.8 \\
\hline \multicolumn{3}{|l|}{ Smoking } \\
\hline Never & 68 & 35.6 \\
\hline Ever & 123 & 64.4 \\
\hline \multicolumn{3}{|l|}{ Pathology } \\
\hline Adenocarcinoma & 79 & 41.4 \\
\hline Squamous & 74 & 38.7 \\
\hline Others & 38 & 19.9 \\
\hline \multicolumn{3}{|l|}{ Location } \\
\hline Central & 49 & 25.7 \\
\hline Peripheral & 142 & 74.3 \\
\hline \multicolumn{3}{|l|}{ Stage } \\
\hline I & 26 & 13.6 \\
\hline II & 10 & 5.2 \\
\hline III & 35 & 18.3 \\
\hline IV & 120 & 62.8 \\
\hline \multicolumn{3}{|l|}{ Systemic therapy } \\
\hline Nothing & 72 & 37.7 \\
\hline Chemotherapy & 70 & 36.6 \\
\hline Targeted therapy & 49 & 25.7 \\
\hline \multicolumn{3}{|l|}{ Origin } \\
\hline Primary & 118 & 61.8 \\
\hline Metastatic & 73 & 38.2 \\
\hline \multicolumn{3}{|l|}{ BED } \\
\hline$\geq 100$ Gy & 152 & 79.6 \\
\hline$<100$ Gy & 39 & 20.4 \\
\hline \multicolumn{3}{|l|}{ Blood group } \\
\hline Type O & 79 & 41.4 \\
\hline Type A & 54 & 28.3 \\
\hline Type B & 39 & 20.4 \\
\hline Type AB & 19 & 9.9 \\
\hline
\end{tabular}

Table 1 (continued)
Table 1 (continued)

\begin{tabular}{lcc}
\hline Characters & Number of cases & Constituent ratio (\%) \\
\hline Pre-treatment CLCs & & \\
$\leq 1.4 \times 10^{9} / \mathrm{L}$ & 100 & 52.4 \\
$>1.4 \times 10^{9} / \mathrm{L}$ & 91 & 47.6 \\
Post-treatment & & \\
CLCs & & \\
$<1.1 \times 10^{9} / \mathrm{L}$ & 92 & 48.2 \\
$\geq 1.1 \times 10^{9} / \mathrm{L}$ & 99 & 51.8 \\
Changes in CLCs & & \\
$\leq 0.3 \times 10^{9} / \mathrm{L}$ & 98 & 51.4 \\
$>0.3 \times 10^{\circ} / \mathrm{L}$ & 93 & 48.7 \\
\hline
\end{tabular}

$\mathrm{BED}$, biological equivalent dose; CLCs, circulating lymphocyte counts.

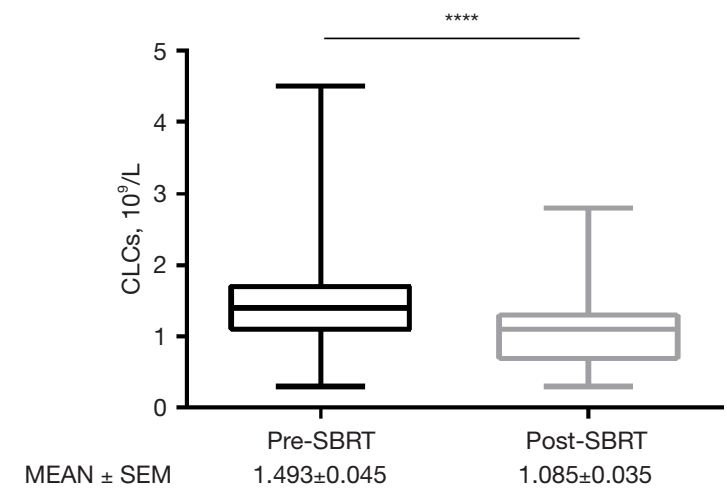

Figure $1 \mathrm{CLCs}$ in the pre-SBRT group and the post-SBRT group. ****, P value $<0.0001$. CLCs, circulating lymphocyte counts; SBRT, stereotactic body radiation therapy; SEM, standard error of mean.

\section{LC correlated with post-SBRT CLCs in lung cancer patients treated with SBRT}

The LC at 1-, 3- and 6-month after SBRT was 97.4\%, $90.6 \%$, and $75.9 \%$, respectively. There was a significant difference in CLCs before and after SBRT (Figure 1). Univariate analysis showed that post-treatment CLCs and gender were correlated with LC at 3- and 6-month after SBRT. Multivariate analysis showed that post-treatment CLCs were independent factors for the optimal short-term efficacy of about 3 and 6 months [hazard ratio $(\mathrm{HR})=0.249$, $95 \%$ confidence interval (CI): 0.067-0.921, P=0.037; $\mathrm{HR}=0.347,95 \%$ CI: $0.152-0.794, \mathrm{P}=0.012]$. The group with higher CLCs after SBRT, which was defined as greater 
than or equal to $1.1 \times 10^{9} / \mathrm{L}$, suggesting better LC (Table 2).

\section{PFS and OS correlated with the ABO blood group in lung cancer patients treated with SBRT}

We used the Kaplan-Meier method to analyze the significance of age, gender, blood type, and CLCs indexes relating to the survival time to judge the prognosis of lung cancer patients. The results are shown in Table 3. Univariate analysis showed that gender, stage, systemic therapy, and blood group were significantly associated with $\mathrm{PFS}(\mathrm{P}<0.05$ for each). Multivariate analysis showed that gender and the type A blood group were independent factors for PFS $(\mathrm{P}<0.05)$. Figure $2 A, 2 B$ showed that the $\mathrm{PFS}$ and OS of A-type blood patients were shorter than those of the non-A-type blood patients (PFS: 6.5 vs. 10 months, HR =1.535, 95\% CI: 1.069 2.204, $\mathrm{P}=0.020$, Table 3; OS: 24 vs. 41 months, $\mathrm{HR}=1.578,95 \%$ CI: $1.003-2.481, \mathrm{P}=0.048$, Table 4). In addition, there were no significant differences between other factors with OS (Table 4).

\section{Post-SBRT CLCs in the non-A blood group correlated with OS of lung cancer patients treated with SBRT}

In the subgroup analysis, univariate and multivariate logistic-regression analyses were used to expound the prognostic factors for OS in A blood group patients or non-A blood group patients. The results are shown in Table 5 . In the non-A blood group, the OS of patients with high post-SBRT CLCs was longer than those with low postSBRT CLCs $(\mathrm{P}<0.05)$. There were no significant prognostic factors in the A blood group. Furthermore, there were no differences in baseline clinical characteristics through the chi-square test between A vs. non-A blood group and high $v s$. low post-SBRT CLCS. The $\mathrm{P}$ values were greater than 0.05 , which indicated that the baseline characteristics between groups were balanced to some extent (Table 6).

\section{PFS and OS prognostic value of post-SBRT CLCs combined with ABO blood groups}

Survival analysis was performed to identify whether a patient's blood group and post-SBRT CLCs exerted combined prognostic influence on the PFS and OS of our patients. The median PFS of non-A-type blood patients with high post-SBRT CLCs was the longest (Figure $3 A$; $\mathrm{P}<0.05)$. As shown in Figure $3 B$, the median OS of patients with non-A blood group and high post-SBRT CLCs was 42 months, while the OS of patients with non-A blood and low post-SBRT CLC, A blood and high post-SBRT CLC, and A blood and low post-SBRT CLC were 32, 23, and 24 months, respectively. Compared with other three groups referenced above, subgroup analysis showed that patients with a non-A blood group and high post-SBRT CLCS had significant differences $(\mathrm{P}=0.04,0.007$, and 0.04 , respectively). There was no statistical difference among the other three groups $(\mathrm{P}>0.05)$.

\section{Discussion}

SBRT is a standard treatment method for early-stage NSCLC patients who are inoperable or who refuse surgery.

CHISEL study [2019] has shown that, compared with conventional radiotherapy, SBRT significantly improved LC and OS (2-year OS was $77 \%$ with SBRT vs. $59 \%$ without SBRT; 2 -year LC was $89 \%$ with SBRT vs. $65 \%$ without SBRT) (2). For stage IV patients, the results of stereotactic ablative radiotherapy for the comprehensive treatment of oligometastases (SABR-COMET), published in The Lancet in 2018, were surprising. Compared with systemic treatment alone, SBRT combined with standard treatment was shown to prolong the median OS from 28 to 41 months, and the PFS time increased from 6 to 12 months (6).

Circulating lymphocytes have a decisive influence on the immune system of cancer patients, and our research found that the number of lymphocytes decreased after SBRT, confirming the high radiosensitivity of these cells, which is consistent with the finding of Rutkowski et al. [2017] (21). Giuliani et al. [2016] found that, in lung cancer SBRT patients, the neutrophil to lymphocyte ratio and monocyte to lymphocyte ratio were independent prognostic factors for OS (22). Similarly, Luo et al. [2018] reported that a high platelet to lymphocyte ratio and a low lymphocyte to monocyte ratio were independently associated with poor OS (23). The increase of post-treatment cytotoxic CD8 ${ }^{+}$ T-cell predicted PFS prolongation in stage I NSCLC patients (24), while peripheral lymphopenia after SBRT predicted poorer outcomes in hepatocellular carcinoma (HCC) patients (7). In our study, post-treatment CLCs correlated with LC of lung cancer SBRT patients. Existing research shows that lymphocyte subsets, including NK cells, T cells, B cells, cytotoxic cells, and helper cells, all participate in the antitumor immune response (25). The number of tumor-infiltrating lymphocytes, which is an important component of the tumor microenvironment (TME), was found to be significantly correlated with enhanced antitumor activity and tumor destruction (26). 


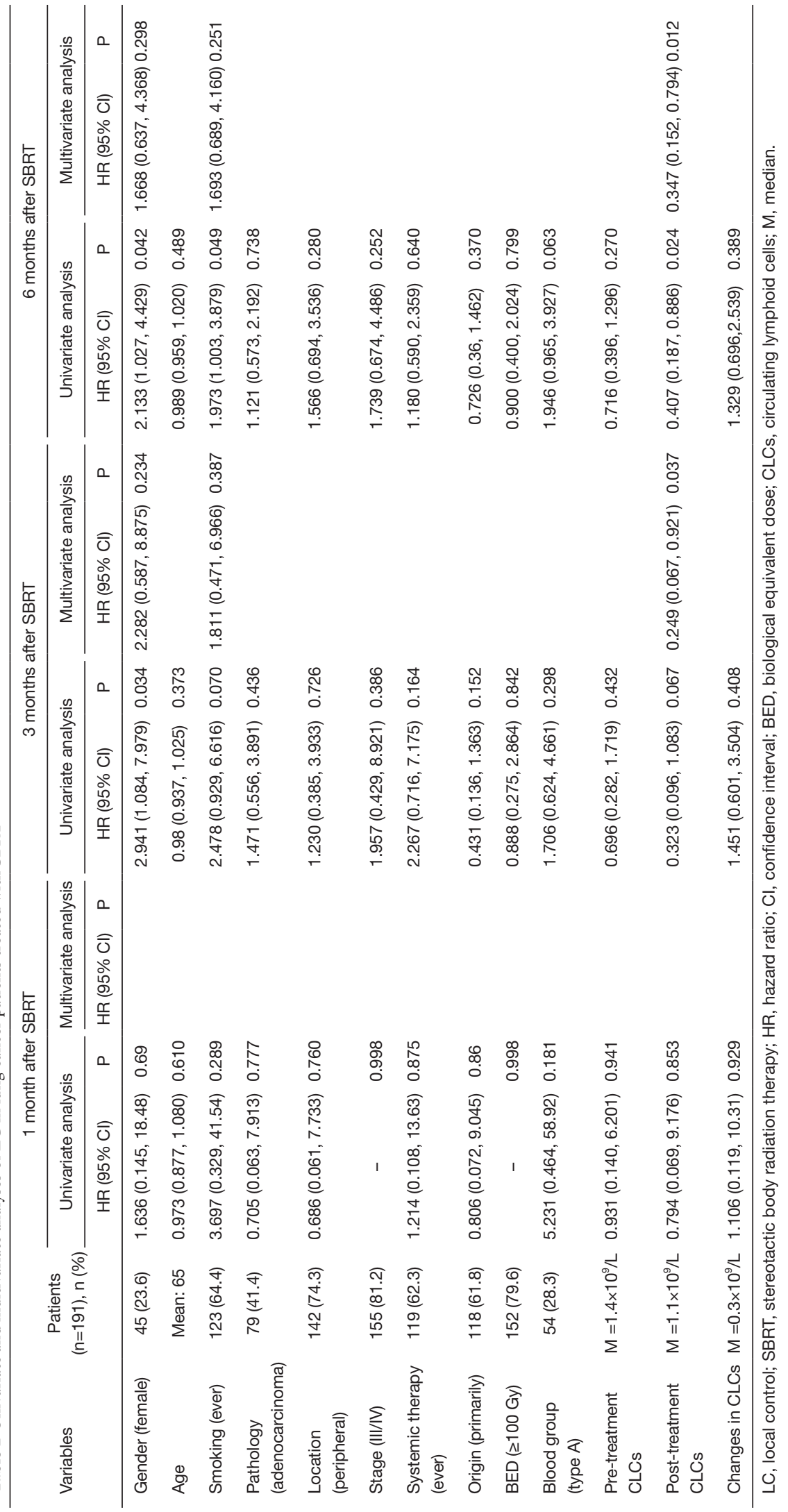


Table 3 Univariate and multivariate analyses of PFS in lung cancer patients treated with SBRT

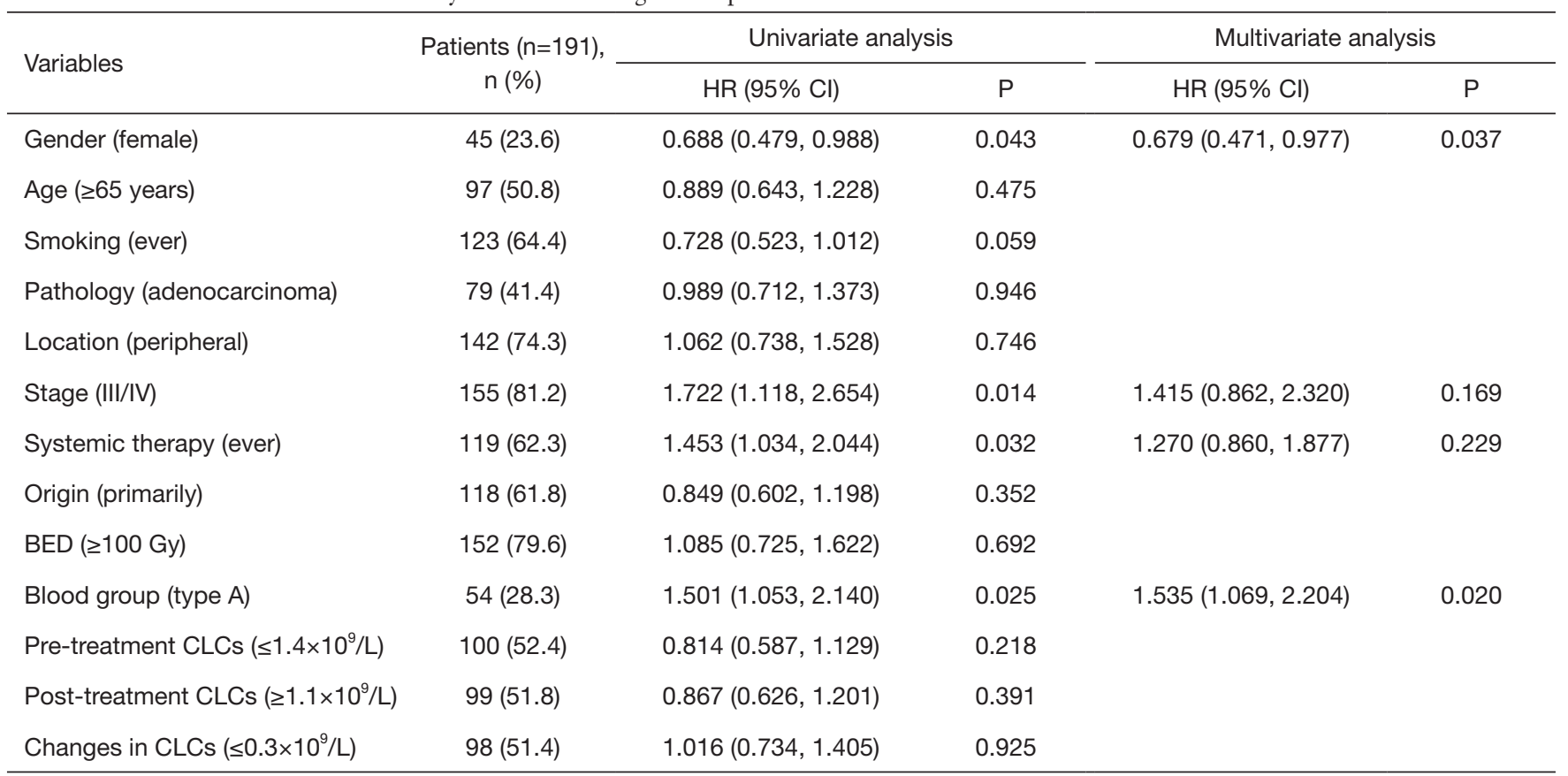

PFS, progression-free survival; SBRT, stereotactic body radiation therapy; HR, hazard ratio; Cl, confidence interval; BED, biological equivalent dose; CLCs, circulating lymphoid cells.
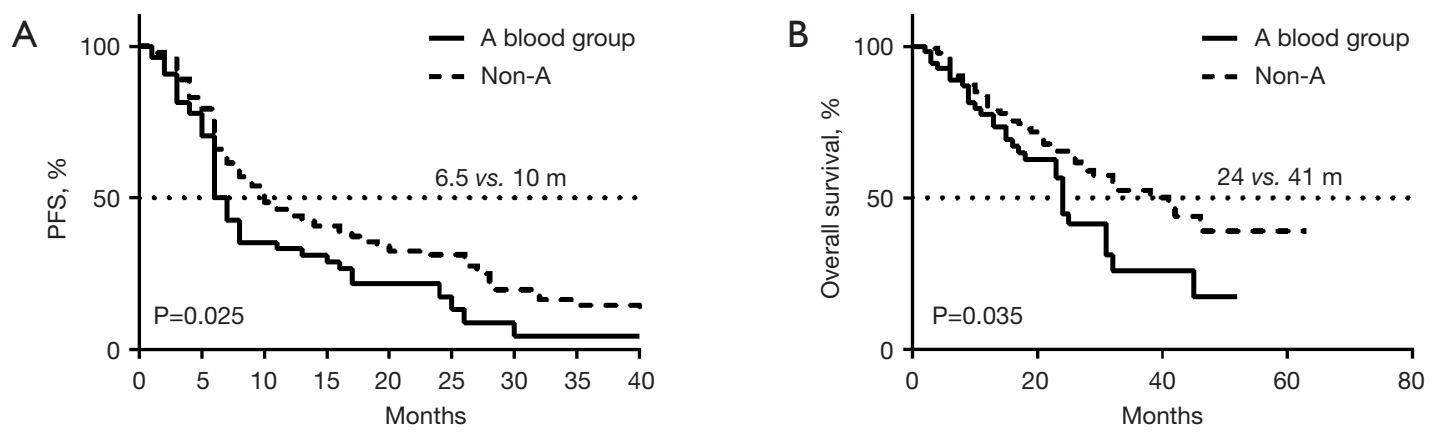

Figure 2 Kaplan-Meier analysis of PFS and OS for all patients grouped by A blood group. (A) The PFS in patients with A blood group and non-A blood group. Non-A: the other three non-A blood groups. (B) The OS in patients with type A blood group and non-A blood group. Non-A: the other three non-A blood groups. PFS, progression-free survival; OS, overall survival; SBRT, stereotactic body radiation therapy; CLCs, circulating lymphocyte counts.

Proinflammatory T-cell increased after SABR, while the number of regulatory $T$-cells decreased, suggesting that SABR can regulate the immune response (21).

Lymphocyte subsets have also been shown to decline after irradiation, but there are significant differences in the decline and recovery rate. The vitro experiments of Falcke [2018] showed that NK and B cells were more sensitive to radiation than other immune cell populations in inducing cell death (27). The studies of Zhuang [2019] and Maehata [2013] both indicated that B cells decreased to a greater extent than NK cells following $\operatorname{SBRT}(7,28)$. This may be because mature $\mathrm{CD} 56^{+} \mathrm{CD} 16^{+} \mathrm{NK}$ cells did not decrease after SBRT, while immature CD56brCD16 ${ }^{-} \mathrm{NK}$ cells decreased significantly (29). The peripheral NK cells, TPLC and CD8 ${ }^{+} \mathrm{T}$ counts were higher in patients with better survival, according to a stratified analysis performed 
Table 4 Univariate and multivariate analyses of OS in lung cancer patients treated with SBRT

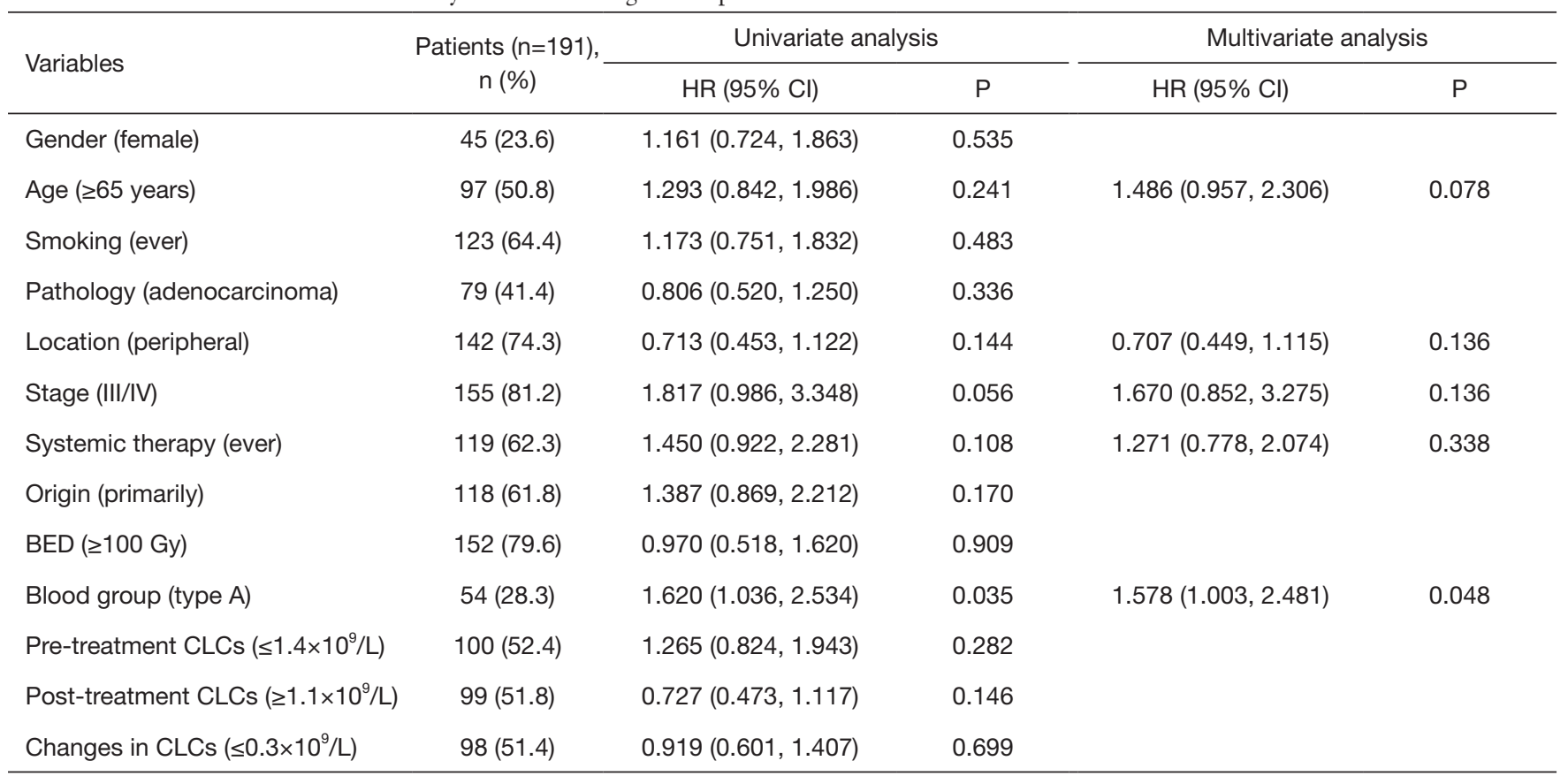

OS, overall survival; SBRT, stereotactic body radiation therapy; HR, hazard ratio; Cl, confidence interval; BED, biological equivalent dose; CLCs, circulating lymphoid cells.

at OS of 2 years, and there was no significant difference in B cells (7). Pervious research also suggested that the direct effect of radiation on lymphocyte count may be the result of apoptosis of mature cells, or the high radiosensitivity of their progenitor stem cells, reduces their bone marrow production (30).

Our findings showed that lymphocyte counts before and after SBRT were not correlated with PFS or OS, while type A patients had poorer PFS and OS than patients with non-A type blood. but the $\mathrm{ABO}$ blood group is an important factor of individual radiosensitivity (31). Our study found that, in lung cancer patients treated with SBRT, patients with the A blood group had the lowest PFS and OS compared with patients with the other three non-A blood groups. In resected NSCLC, Fukumoto et al. [2015] studied 333 patients and found that the 5-year OS and PFS of patients with type A blood were the lowest (only $67.2 \%$ and $62.3 \%$, respectively), while the 5-year OS and PFS of patients with type O blood group were $83.0 \%$ and $71.6 \%$, respectively (32). Li et al. [2015] found similar results (33). Compared with type A or type $\mathrm{AB}$ patients, the $\mathrm{OS}$ of type $\mathrm{O}$ or type $\mathrm{B}$ patients was significantly prolonged (74.9 months for type $\mathrm{O}$ and $\mathrm{B}$ patients $v s$. 61.5 months for type $\mathrm{A}$ and type $\mathrm{AB}$ patients). However, the mechanism behind this is not clear, and there are several possible hypotheses at present.

The expression of blood group antigen $\mathrm{A} / \mathrm{B}$ on cancer cells is regulated by hypermethylation of the $\mathrm{ABO}$ gene promoter. The loss of blood group antigen $\mathrm{A}$ has been found to be related to the bronchial carcinogenesis and the prognosis of NSCLC $(34,35)$. We detected that, in the non-A blood group, high post-SBRT CLCs patients had longer OS than low post-SBRT CLC patients. The single nucleotide polymorphism of the $\mathrm{ABO}$ was related to the circulating level of inflammatory molecules, such as selectin and soluble intracellular adhesion molecule-1 (36). These inflammatory molecules are related to angiogenesis, proliferation, invasion, and migration of tumors. Although Elahimanesh [2013] and Habibi [2016] reported that the radiosensitivity of A+ blood was the highest $(31,37)$. Van Alsten et al. [2021] found that individuals with the type A blood group had the lowest levels of all ABO-associated inflammation markers, such as sVEGFR2, sVEGFR3, and sGP130 (38). Viswanathan conducted a proliferation experiment of lymphocytes of different blood types in vitro and found that, after receiving 4 Gy of radiation, the number of lymphocytes in patients with type A blood decreased the greatest amount, followed by the patients with type O blood (39). These show that type A blood is 


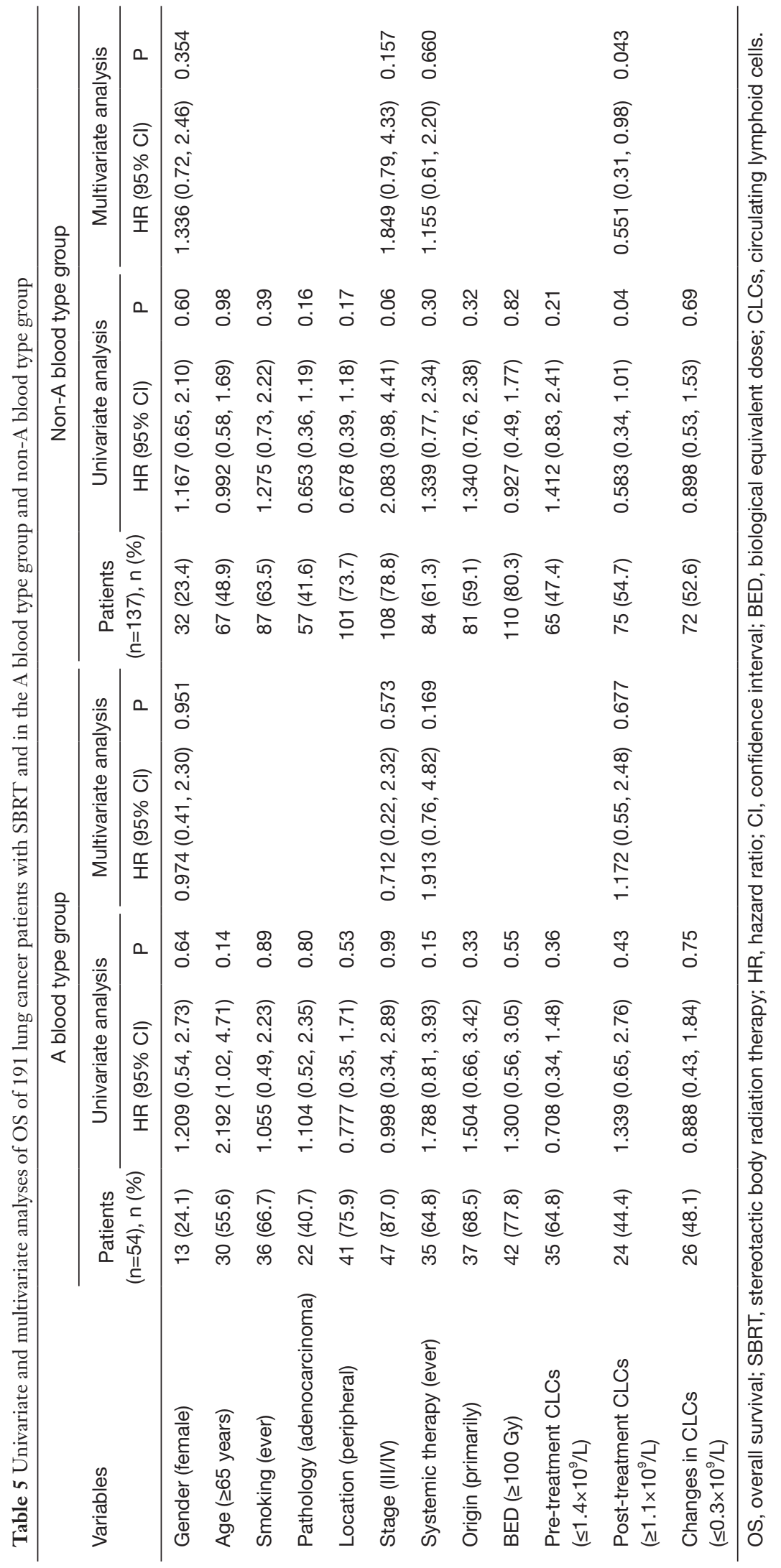


Table 6 Chi-square test of baseline clinical characteristics of patients grouped by blood group and post-SBRT CLCs medians

\begin{tabular}{|c|c|c|c|c|c|c|}
\hline Variables & \multicolumn{3}{|c|}{ Blood group } & \multicolumn{3}{|c|}{ Post-SBRT CLCs } \\
\hline Gender (female) & $13(24.1)$ & $32(23.4)$ & 0.916 & $24(24.2)$ & $21(22.8)$ & 0.818 \\
\hline Age ( $\geq 65$ years) & $30(55.6)$ & $67(48.9)$ & 0.408 & $52(52.5)$ & $45(48.9)$ & 0.618 \\
\hline Location (peripheral) & $41(75.9)$ & $101(73.7)$ & 0.754 & $76(76.8)$ & $66(71.7)$ & 0.427 \\
\hline Stage (III/IV) & $47(87.0)$ & $108(78.8)$ & 0.192 & 79 (79.8) & $76(82.6)$ & 0.620 \\
\hline Systemic therapy (ever) & $35(64.8)$ & $84(61.3)$ & 0.653 & $67(67.7)$ & $52(56.5)$ & 0.112 \\
\hline
\end{tabular}

SBRT, stereotactic body radiation therapy; CLCs, circulating lymphoid cells; BED, biological equivalent dose.
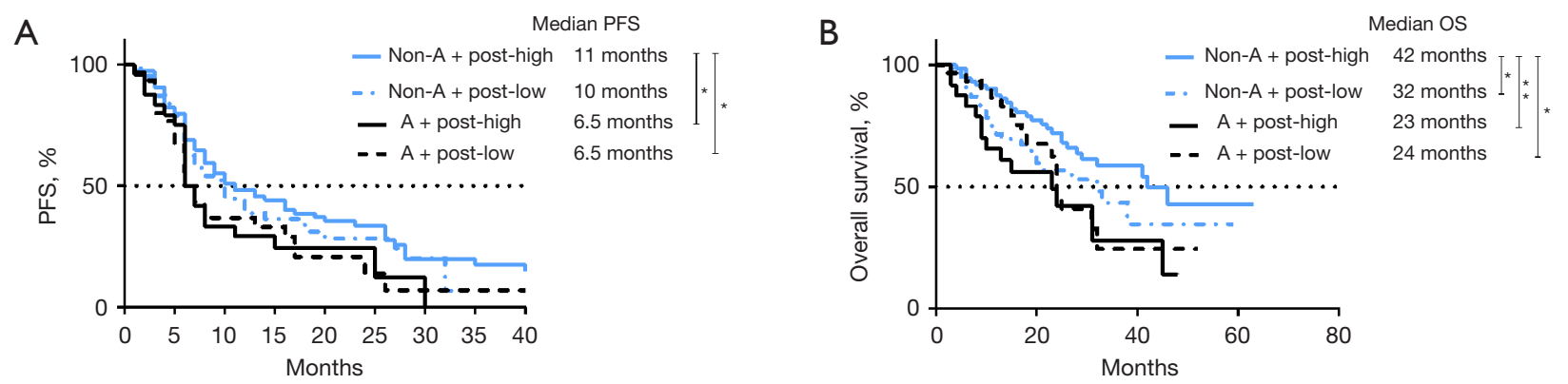

Figure 3 Log-rank test of PFS and OS for all patients grouped by A blood group and post-SBRT CLCs. (A) The PFS in the non-A blood and high post-SBRT CLCs group, the non-A blood and low post-SBRT CLC group, the A blood and high post-SBRT CLC group, and the A blood and low post-SBRT CLC group. (B) The OS in the non-A blood and high post-SBRT CLCs group, the non-A blood and low postSBRT CLC group, the A blood and high post-SBRT CLC group, and the A blood and low post-SBRT CLC group. *, P value <0.05; **, $\mathrm{P}$ value $<0.01$. PFS, progression-free survival; OS, overall survival; SBRT, stereotactic body radiation therapy; CLCs, circulating lymphocyte counts.

more affected by radiotherapy, but its immune environment is cold, which may limit the ability of lymphocytes to build immunity after radiotherapy. In the non-A blood group, the immune environment is hot, and lymphocytes can better reflect the reserve level of immune function after treatment.

However, our retrospective observational study had some limitations. Advanced patients (III/IV) were included in this study. Distant metastasis and non-pulmonary death were the main causes of progression and death, which may have led to low PFS and OS time. SBRT combined with systemic therapy (mainly chemotherapy and targeted therapy) was most used. Furthermore, subgroups had different clinical features. Even if the clinical features of each group were balanced in subgroup analysis, the effects of PFS, OS, and LC are more complicated in real world. Our research attempted to provide a simple and feasible prediction method for clinical use that could be helpful for further screening. Additionally, future welldesigned prospective studies are needed to further investigate the relationship between CLCs, including lymphocyte subgroups, and the prognosis of lung cancer SBRT patients.

\section{Conclusions}

Post-treatment CLCs were correlated with LC at 3- and 
6-month after SBRT. Lung cancer SBRT patients with high-post-SBRT CLCs and non-A blood groups seem to exhibits best PFS and OS, which represent a potential opportunity to improve the clinical management of these patients. The potential mechanisms, which may be associated with immunity, warrants further investigation.

\section{Acknowledgments}

Funding: This work was supported in part by the Chinese National Science Foundation Projects (Nos. NSFC 81874221 and 81872458) and the Science and Technology Agency of Taizhou City (No. 20ywa09), China.

\section{Footnote}

Reporting Checklist: The authors have completed the REMARK reporting checklist. Available at https://jtd. amegroups.com/article/view/10.21037/jtd-22-130/rc

Data Sharing Statement: Available at https://jtd.amegroups. com/article/view/10.21037/jtd-22-130/dss

Conflicts of Interest: All authors have completed the ICMJE uniform disclosure form (available at https://jtd.amegroups. com/article/view/10.21037/jtd-22-130/coif). The authors have no conflicts of interest to declare.

Ethical Statement: The authors are accountable for all aspects of the work in ensuring that questions related to the accuracy or integrity of any part of the work are appropriately investigated and resolved. The study was conducted in accordance with the Declaration of Helsinki (as revised in 2013). This retrospective study was approved by the ethics committee of Taizhou Hospital of Zhejiang Province (No. K20170320) and individual consent for this retrospective analysis was waived.

Open Access Statement: This is an Open Access article distributed in accordance with the Creative Commons Attribution-NonCommercial-NoDerivs 4.0 International License (CC BY-NC-ND 4.0), which permits the noncommercial replication and distribution of the article with the strict proviso that no changes or edits are made and the original work is properly cited (including links to both the formal publication through the relevant DOI and the license). See: https://creativecommons.org/ licenses/by-nc-nd/4.0/.

\section{References}

1. Sung H, Ferlay J, Siegel RL, et al. Global Cancer Statistics 2020: GLOBOCAN Estimates of Incidence and Mortality Worldwide for 36 Cancers in 185 Countries. CA Cancer J Clin 2021;71:209-49.

2. Ball D, Mai GT, Vinod S, et al. Stereotactic ablative radiotherapy versus standard radiotherapy in stage 1 nonsmall-cell lung cancer (TROG 09.02 CHISEL): a phase 3 , open-label, randomised controlled trial. Lancet Oncol 2019;20:494-503.

3. von Reibnitz D, Shaikh F, Wu AJ, et al. Stereotactic body radiation therapy (SBRT) improves local control and overall survival compared to conventionally fractionated radiation for stage I non-small cell lung cancer (NSCLC). Acta Oncol 2018;57:1567-73.

4. Timmerman RD, Hu C, Michalski JM, et al. Longterm Results of Stereotactic Body Radiation Therapy in Medically Inoperable Stage I Non-Small Cell Lung Cancer. JAMA Oncol 2018;4:1287-8.

5. Chang JY, Senan S, Paul MA, et al. Stereotactic ablative radiotherapy versus lobectomy for operable stage I nonsmall-cell lung cancer: a pooled analysis of two randomised trials. Lancet Oncol 2015;16:630-7.

6. Palma DA, Olson R, Harrow S, et al. Stereotactic ablative radiotherapy versus standard of care palliative treatment in patients with oligometastatic cancers (SABRCOMET): a randomised, phase 2, open-label trial. Lancet 2019;393:2051-8.

7. Zhuang Y, Yuan BY, Chen GW, et al. Association Between Circulating Lymphocyte Populations and Outcome After Stereotactic Body Radiation Therapy in Patients With Hepatocellular Carcinoma. Front Oncol 2019;9:896.

8. Wu M, Yang S, Feng X, et al. Prognostic value of the postoperative neutrophil-lymphocyte ratio in solid tumors: A meta-analysis. PLoS One 2021;16:e250091.

9. Durdik M, Kosik P, Jakl L, et al. Imaging flow cytometry and fluorescence microscopy in assessing radiation response in lymphocytes from umbilical cord blood and cancer patients. Cytometry A 2021;99:1198-208.

10. Afghahi A, Mathur M, Seto T, et al. Lymphopenia after adjuvant radiotherapy (RT) to predict poor survival in triple-negative breast cancer (TNBC). J Clin Oncol 2015;33:abstr 1069.

11. Damen PJJ, Kroese TE, van Hillegersberg R, et al. The Influence of Severe Radiation-Induced Lymphopenia on Overall Survival in Solid Tumors: A Systematic Review and Meta-Analysis. Int J Radiat Oncol Biol Phys 
2021;111:936-48.

12. Campian JL, Sarai G, Ye X, et al. Association between severe treatment-related lymphopenia and progressionfree survival in patients with newly diagnosed squamous cell head and neck cancer. Head Neck 2014;36:1747-53.

13. Bradley JD, Hu C, Komaki RR, et al. Long-Term Results of NRG Oncology RTOG 0617: Standard- Versus HighDose Chemoradiotherapy With or Without Cetuximab for Unresectable Stage III Non-Small-Cell Lung Cancer. J Clin Oncol 2020;38:706-14.

14. Upadhyay R, Venkatesulu BP, Giridhar P, et al. Risk and impact of radiation related lymphopenia in lung cancer: A systematic review and meta-analysis. Radiother Oncol 2021;157:225-33.

15. Jin JY, Gu A, Wang W, et al. Ultra-high dose rate effect on circulating immune cells: A potential mechanism for FLASH effect? Radiother Oncol 2020;149:55-62.

16. McLaughlin MF, Alam M, Smith L, et al. Stereotactic body radiation therapy mitigates radiation induced lymphopenia in early stage non-small cell lung cancer. PLoS One 2020;15:e0241505.

17. Shen Y, Meng Y, Tang X, et al. P1. 04-69 Modelling the Immunosuppressive Difference of SBRT and CRT by Simulating the Dose to Circulating Lymphocytes in NonSmall Cell Lung Cancer. J Thorac Oncol 2019;14:S468.

18. Mao Y, Yang W, Qi Q, et al. Blood groups A and AB are associated with increased gastric cancer risk: evidence from a large genetic study and systematic review. BMC Cancer 2019;19:164.

19. Groot HE, Villegas Sierra LE, Said MA, et al. Genetically Determined ABO Blood Group and its Associations With Health and Disease. Arterioscler Thromb Vasc Biol 2020;40:830-8.

20. Liu F, Li C, Zhu J, et al. ABO blood type and risk of hepatocellular carcinoma: a meta-analysis. Expert Rev Gastroenterol Hepatol 2018;12:927-33.

21. Rutkowski J, Ślebioda T, Kmieć Z, et al. Changes in systemic immune response after stereotactic ablative radiotherapy. Preliminary results of a prospective study in patients with early lung cancer. Pol Arch Intern Med 2017;127:245-53.

22. Giuliani M, Sampson LR, Wong O, et al. Prognostic value of pretreatment circulating neutrophils, monocytes, and lymphocytes on outcomes in lung stereotactic body radiotherapy. Curr Oncol 2016;23:e362-8.

23. Luo H, Ge H, Cui Y, et al. Systemic Inflammation Biomarkers Predict Survival in Patients of Early Stage Non-Small Cell Lung Cancer Treated With Stereotactic
Ablative Radiotherapy - A Single Center Experience. J Cancer 2018;9:182-8.

24. Zheng Y, Shi A, Wang W, et al. Posttreatment Immune Parameters Predict Cancer Control and Pneumonitis in Stage I Non-Small-Cell Lung Cancer Patients Treated With Stereotactic Ablative Radiotherapy. Clin Lung Cancer 2018;19:e399-404.

25. Basu A, Ramamoorthi G, Albert G, et al. Differentiation and Regulation of TH Cells: A Balancing Act for Cancer Immunotherapy. Front Immunol 2021;12:669474.

26. Labani-Motlagh A, Ashja-Mahdavi M, Loskog A. The Tumor Microenvironment: A Milieu Hindering and Obstructing Antitumor Immune Responses. Front Immunol 2020;11:940.

27. Falcke SE, Rühle PF, Deloch L, et al. Clinically Relevant Radiation Exposure Differentially Impacts Forms of Cell Death in Human Cells of the Innate and Adaptive Immune System. Int J Mol Sci 2018;19:3574.

28. Maehata Y, Onishi H, Kuriyama K, et al. Immune responses following stereotactic body radiotherapy for stage I primary lung cancer. Biomed Res Int 2013;2013:731346.

29. Gustafson MP, Bornschlegl S, Park SS, et al. Comprehensive assessment of circulating immune cell populations in response to stereotactic body radiation therapy in patients with liver cancer. Adv Radiat Oncol 2017;2:540-7.

30. Kapoor V, Collins A, Griffith K, et al. Radiation induces iatrogenic immunosuppression by indirectly affecting hematopoiesis in bone marrow. Oncotarget 2020;11:1681-90.

31. Elahimanesh F, Shabestani Monfared A, Khosravifarsani M, et al. Is Radiosensitivity Associated to Different Types of Blood Groups? (A cytogenetic study). Int J Mol Cell Med 2013;2:131-5.

32. Fukumoto K, Taniguchi T, Usami N, et al. The ABO blood group is an independent prognostic factor in patients with resected non-small cell lung cancer. J Epidemiol 2015;25:110-6.

33. Li N, Xu M, Li CF, et al. Prognostic role of the ABO blood types in Chinese patients with curatively resected non-small cell lung cancer: a retrospective analysis of 1601 cases at a single cancer center. Chin J Cancer 2015;34:475-82.

34. León-Atance P, Moreno-Mata N, González-Aragoneses F, et al. Prognostic influence of loss of blood group A antigen expression in pathologic stage I non-small-cell lung cancer. Arch Bronconeumol 2012;48:49-54. 
35. Lee JS, Ro JY, Sahin AA, et al. Expression of blood-group antigen A--a favorable prognostic factor in non-small-cell lung cancer. N Engl J Med 1991;324:1084-90.

36. Sliz E, Kalaoja M, Ahola-Olli A, et al. Genome-wide association study identifies seven novel loci associating with circulating cytokines and cell adhesion molecules in Finns. J Med Genet 2019;56:607-16.

37. Habibi M, Namimoghadam A, Korouni R, et al. Radiationinduced nausea and vomiting: Is ABO blood group as important as radiation and patient-related factors? An

Cite this article as: Chen M, Chen K, Li S, Meng Y, Shi Y, Chen X, Yang H. The prognostic value of circulating lymphocyte counts and ABO blood group in lung cancer stereotactic body radiation therapy: a retrospective study. J Thorac Dis 2022;14(2):494-506. doi: 10.21037/jtd-22-130 observational study. Medicine (Baltimore) 2016;95:e4334.

38. Van Alsten SC, Aversa JG, Santo L, et al. Association between $\mathrm{ABO}$ and Duffy blood types and circulating chemokines and cytokines. Genes Immun 2021;22:161-71.

39. Viswanathan S, Kanagaraj K, Raavi V, et al. Does proliferation capacity of lymphocytes depend on human blood types? J Cell Biochem 2019;120:5722-8.

(English Language Editor: C. Mullens) 